\title{
ESTATÍSTICAS AMBIENTAIS DE ÁGUA A PARTIR DA APLICAÇÃo DE COEFICIENTES TÉCNICOS - UM ESTUDO DE CASO
}

\author{
Rodrigo da Silveira Pereira e Julio Jorge Gonçalves da Costa \\ Instituto Brasileiro de Geografia e Estatística, Brasil \\ rodrigopereirageo@gmail.com
}

\begin{abstract}
: este estudo de caso consiste na aplicação de coeficientes técnicos nas estatísticas oficiais de produção industrial brasileira (2010), de forma a converte estatísticas econômicas em ambientais (recurso natural água). Ao aplicar os coeficientes máximos, as estatísticas de água obtidas para retirada, consumo e efluentes foram de 6,5, 2,7 e 4,1 bilhões de $m^{3}$ respectivamente. Os resultados obtidos sugerem que a metodologia empregada é útil para e gestão dos recursos hídricos. Entretanto possui limitações importantes tais como significativas amplitudes observadas nos coeficientes e a reduzida quantidade de produtos contemplados, 27,7\% do total de classes integrantes das estatísticas oficiais da produção industrial no Brasil em 2010. Tais limitações podem ser atenuadas pela aplicação dos princípios de educação ambiental na produção de estatísticas de água em bacias hidrográficas, integrando sociedade e instituições de ensino.
\end{abstract}

\section{INTRODUÇÃO}

A produção de estatísticas ambientais no Brasil apresenta muitas lacunas. Estas podem ser associadas com a natureza das pesquisas em meio ambiente. A aplicação de questionários para a obtenção direta de dados, bem como a utilização de registros administrativos, técnicas consagradas no âmbito das pesquisas estatísticas socioeconômicas, encontra pouca aplicabilidade nas pesquisas ambientais. Um dos principais problemas na obtenção por métodos diretos está na qualidade da informação ambiental, com especificidades inerentes às variações no tempo e no espaço. A elaboração de um questionário para obtenção de estatísticas ambientais, eficiente, requer um esforço de preparação que pode representar um alto custo para as instituições demandantes, por exemplo, os institutos de estatísticas oficiais.

A partir deste problema, tendo em vista que os recursos naturais integram a matriz de produção agropecuária e industrial como insumos, bem como a necessidade de se avaliar o uso dos recursos naturais no Brasil, a aplicação de constantes de conversão para mensurar o montante de recursos naturais utilizada constitui-se em importante meio para a obtenção de estatísticas. Um recente trabalho desenvolvido por um convênio composto pela Fundação de Apoio à Universidade Federal de Viçosa - FUNARBE, Ministério do Meio Ambiente - MMA e Fundação Banco do Brasil - FBB, apresenta como resultados estas constantes de conversão, definidas como coeficientes técnicos para utilização de recursos hídricos nas atividades industriais e agricultura irrigada (FUNARBE, 2011).

Considerando o problema acima exposto e as possibilidades de obtenção indireta de estatísticas ambientais através de constantes de conversão, este trabalho tem como objetivo converter, empiricamente, estatísticas econômicas em ambientais, especificamente, estatísticas do recurso natural água. Adicionalmente, é apresentada uma discussão sobre limitações ao uso dos coeficientes técnicos às estatísticas oficiais da produção industrial no Brasil, bem como de que forma, a educação ambiental pode contribuir para a construção de estatísticas de recursos hídricos, no âmbito das bacias hidrográficas.

\section{REVISÃO BIBLIOGRÁFICA}

No Brasil, as estatísticas de estoque de recursos hídricos são os principais resultados apresentados pelo relatório Conjuntura dos Recursos Hídricos no Brasil, produzido pela Agência Nacional de Águas (ANA). Pesquisas produzidas pelo Instituto Brasileiro de Geografia e Estatística (IBGE) fornecem um panorama da situação dos recursos hídricos no país sob a ótica dos domicílios, por recorte político-administrativo. Dentre essas, destacam-se as seguintes:

- Pesquisa Nacional de Saneamento Básico - PNSB;

- Pesquisa Nacional por Amostra de Domicílios - PNAD;

- Censo Demográfico.

In: M.A. Sorto (Ed.), Advances in statistics education: developments, experiences and assessments. Proceedings of the Satellite conference of the International Association for Statistical Education (IASE), July 2015, Rio de Janeiro, Brazil. 
Em termos internacionais, a produção bibliográfica para estatísticas ambientais e métodos de obtenção é restrita. Esta é atribuída, principalmente, à divisão de estatísticas da Organização das Nações Unidas (UNSD), por meio de diretrizes e manuais para a coleta e produção. Destacam-se:

- Indicators of Sustainable Development: Guidelines and Methodologies;

- International Recommendations for Water Statistics;

- System of Environmental Economic Accounts;

- System of Environmental Economic Accounts - Water;

- Framework for the Development of Environment Statistics.

\section{Sobre a Matriz de Coeficientes Técnicos}

Coeficientes técnicos de utilização de recursos hídricos na atividade industrial constituem objeto de estudo de Lisboa (2010) e Lisboa et al (2014). Nestes estudos são apresentadas todas as potencialidades proporcionadas pela matriz, bem como todo o arcabouço teórico utilizado em sua elaboração. Segundo estes autores, as informações sobre as vazões de retirada, consumo e efluentes foram obtidas por tipologias industriais, de acordo com o sistema nacional de classificação das atividades econômicas - CNAE, tabuladas, analisadas quanto às dispersões em função de especificidades nos processos produtivos, validadas a partir de comparações com referências da Agência Nacional de Águas - ANA e informações dos setores produtivos. Ainda de acordo com estes autores, o estudo engloba 33 tipologias industriais que são as mais representativas no âmbito da produção industrial doméstica.

A versão da matriz de coeficientes técnicos utilizada neste estudo de caso está contida no documento Relatório Final dos Coeficientes Técnicos de Recursos Hídricos das Atividades Industriais e Agricultura Irrigada (FUNARBE, 2011). Segundo esta referência, 3.786 empresas foram analisadas quanto à utilização de recursos hídricos (retirada, consumo e efluentes), sendo que 3.631 (95,9\% do total) não possuíam dados ou não se enquadravam nas tipologias de interesse do estudo. Para algumas empresas, as informações foram incompletas, sendo disponibilizado apenas um dos coeficientes. Em relação aos dados utilizados para a construção da matriz, cerca de $50 \%(47,7 \%)$ destes foram obtidos de forma indireta, através de registros administrativos das empresas pesquisadas. Parte destes dados foi obtida através de estudos de caso. Este estudo, tendo como base para a definição do escopo das atividades industriais pesquisadas a Classificação Nacional de Atividades Econômicas - CNAE 2.0 (IBGE, 2010a), apresenta os coeficientes ao nível de classe. (FUNARBE, op. cit.).

\section{METODOLOGIA}

A metodologia empregada neste estudo de caso foi direcionada de forma a permitir a aplicação direta dos coeficientes que compõem a matriz de coeficientes técnicos. Dessa forma, foram desenvolvidas as seguintes etapas:

- Compatibilização entre as codificações utilizadas nas estatísticas econômicas da produção industrial para o ano de 2010 (IBGE, 2010b). Estas estatísticas são apresentadas em códigos conforme a Lista de Produtos da Indústria - PRODLIST 2010 (IBGE, 2010c);

- Correlação entre PRODLIST e CNAE 2.0, para divisão, grupo, classe, com o devido enquadramento dos produtos segundo a codificação PRODLIST.

Após as etapas anteriores, foi feita a verificação das unidades de medida do montante da produção por código PRODLIST, de forma colocar o montante da produção e coeficientes técnicos de retirada, consumo e efluentes sob as mesmas unidades. As classes que não apresentaram unidades correlatas foram excluídas do estudo de caso.

Em alguns coeficientes são verificadas amplitudes significativas. Para minimizar esta limitação, bem como simular uma situação de maior incremento na utilização de recursos hídricos, adotaram-se os valores máximos para os coeficientes como padrão para o estudo de caso.

Por último, foram multiplicados os montantes da produção para o ano, pelos coeficientes de retirada, consumo e efluentes, objetivando uma aproximação do uso da água dentro do setor institucional indústria. 


\section{RESULTADOS}

$\mathrm{Na}$ tabela 1 constam os resultados da compatibilização entre as classificações CNAE 2.0 e PRODLIST que definem o escopo do estudo de caso. Na tabela 2 estão identificados os maiores e menores volumes de retirada, consumo e efluentes por estrutura da CNAE 2.0. Na tabela 3 estão os resultados consolidados para a produção industrial brasileira no ano de 2010, sendo que, nestes resultados, adotou-se como hipótese que o total de retirada é a soma dos totais consumidos e de efluentes. As tabelas citadas são apresentadas a seguir.

Tabela 1: Estatísticas totais de tipologias industriais e quantidade com unidades compatíveis para a conversão em estatísticas de uso da água por estrutura da CNAE 2.0 e Matriz de Coeficientes Técnicos - 2010.

\begin{tabular}{l|c|c|c}
\hline \multicolumn{1}{c|}{ Estrutura CNAE 2.0 } & Quantidade & Quantidade com unidades compatíveis & $\%$ \\
\hline Divisões & 27 & 13 & $48,1 \%$ \\
\hline Grupos & 105 & 30 & $28,6 \%$ \\
\hline Classes & 253 & 70 & $27,7 \%$ \\
\hline
\end{tabular}

Tabela 2: Maiores e menores volumes de retirada, consumo e efluentes por estrutura da CNAE 2.0 $-2010$.

\begin{tabular}{c|c|c|c|c|c|c}
\hline \multirow{2}{*}{$\begin{array}{c}\text { Estrutura } \\
\text { CNAE }\end{array}$} & \multicolumn{3}{|c}{ Maiores volumes $\left(\mathrm{m}^{3}\right)$} & \multicolumn{3}{c}{ Menores volumes $\left(\mathrm{m}^{3}\right)$} \\
\cline { 2 - 7 } Divisão & Retirada & Consumo & Efluentes & Retirada & Consumo & Efluentes \\
& $1,9 \times 10^{9}$ & $(10)$ & $(20)$ & $(14)$ & $(14)$ & $(14)$ \\
& $(202)$ & $(072)$ & $(202)$ & $(142)$ & $(142)$ & $(142)$ \\
\hline Grupo & $1,5 \times 10^{9}$ & $5,4 \times 10^{8}$ & $1,1 \times 10^{9}$ & $3,9 \times 10^{3}$ & $7,4 \times 10^{3}$ & $3,1 \times 10^{4}$ \\
\hline Classe & $(2029)$ & $(0729)$ & $(2029)$ & $(1422)$ & $(1422)$ & $(0721)$ \\
& $1,1 \times 10^{9}$ & $4,0 \times 10^{8}$ & $7,9 \times 10^{8}$ & $1,6 \times 10^{3}$ & $3,1 \times 10^{3}$ & $7,3 \times 10^{3}$ \\
\hline
\end{tabular}

Nota: Entre parênteses os códigos CNAE correspondentes a cada valor calculado de vazão.

Tabela 3: Volumes absolutos máximos de retirada, consumo e efluentes e diferenças por total de classes de produtos com compatibilidade de unidades para conversão - 2010 .

\begin{tabular}{c|c|c|c|c}
\hline \multicolumn{3}{c|}{ Volumes Totais $\left(\mathrm{m}^{3}\right)$} & $\begin{array}{c}\text { (Consumo }+ \\
\text { Efluentes }) \mathrm{em} \mathrm{m}^{3}\end{array}$ & $\begin{array}{c}\text { Retirada }-(\text { Consumo } \\
+ \text { Efluentes }) \mathrm{em} \mathrm{m}^{3}\end{array}$ \\
\cline { 1 - 2 } Retirada & Consumo & Efluentes & $6,8 \times 10^{9}$ & $-0,3 \times 10^{9}$ \\
\hline $6,5 \times 10^{9}$ & $2,7 \times 10^{9}$ & $4,1 \times 10^{9}$ & \\
\hline
\end{tabular}

\section{DISCUSSÕES}

De acordo com os resultados obtidos para a compatibilização, os coeficientes técnicos da matriz são diretamente aplicáveis em $27,7 \%$ das classes, contemplando aproximadamente 484 produtos com estatísticas produzidas $(20,7 \%)$. As tipologias mais intensivas em uso de recursos hídricos são as de fabricação de produtos químicos, especificamente industriais de fabricação de produtos químicos orgânicos. Em relação a consumo são verificadas ainda as indústrias de produtos alimentícios e as de extração de minerais metálicos, especificamente as indústrias de extração de minerais metálicos não ferrosos. As tipologias menos intensivas são predominantemente as indústrias de confecção de artigos do vestuário e acessórios, especificamente as de fabricação de artigos de malharia.

Quando observados os resultados consolidados sob a hipótese considerada, houve 65 resultados diferentes de zero para a equação "Retirada - (Consumo + Efluentes)", o que corresponde a $13,4 \%$ das estatísticas obtidas. Destes valores, em 54 os valores de retirada são inferiores à soma da água para consumo e a água destinada aos efluentes.

É oportuna a comparação entre os resultados obtidos com os dados de estoques de recursos hídricos do país. A tabela abaixo apresenta a capacidade total de armazenamento dos reservatórios. 
Tabela 4: Capacidade de armazenamento por região hidrográfica $(\mathrm{RH})$

\begin{tabular}{|c|c|}
\hline Região hidrográfica & Capacidade de armazenamento $\left(\mathrm{m}^{3}\right)$ \\
\hline Amazônica & $2,1 \times 10^{10}$ \\
\hline Atlântico Leste & $1,4 \times 10^{10}$ \\
\hline Atlântico Nordeste Ocidental & - \\
\hline Atlântico Nordeste Oriental & $2,6 \times 10^{10}$ \\
\hline Atlântico Sudeste & $1,1 \times 10^{10}$ \\
\hline Atlântico Sul & $1,5 \times 10^{11}$ \\
\hline Paraguai & $7,5 \times 10^{09}$ \\
\hline Paraná & $2,5 \times 10^{11}$ \\
\hline Parnaíba & $7,5 \times 10^{09}$ \\
\hline São Francisco & $7,4 \times 10^{10}$ \\
\hline Tocantins-Araguaia & $1,2 \times 10^{11}$ \\
\hline Uruguai & $1,3 \times 10^{10}$ \\
\hline Total & $6,9 \times 10^{11}$ \\
\hline
\end{tabular}

Fonte: Modificado de ANA (2012).

De acordo com a tabela 4, no Brasil, os reservatórios têm a capacidade total de armazenamento de $6,9 \times 10^{11} \mathrm{~m}^{3}$. O volume total retirado, de acordo com os resultados obtidos, corresponde a menos de $1 \%$ deste valor. Considerando a capacidade total dos reservatórios das áreas com maior concentração de parques industriais $-2,6 \times 10^{11} \mathrm{~m}^{3}$ (regiões hidrográficas Paraná e Atlântico Leste), o volume total retirado passa a corresponder a $2,5 \%$ do volume concentrado em reservatórios.

Os resultados obtidos neste estudo de caso possuem importantes limitações. Para atenuálas, devem ser consideradas, para efeitos de gestão dos recursos hídricos, diferenciações espaciais (diversidade de disponibilidade e de uso da água). No Brasil, a gestão de recursos hídricos é regulada pela Lei Federal $n^{\circ}$ 9.433, Política Nacional de Recursos Hídricos (BRASIL, 1997). Este dispositivo legal prevê, no seu Artigo $1^{\circ}$, que a bacia hidrográfica é a unidade territorial de gestão dos recursos hídricos e implantação da política de recursos hídricos, e que esta gestão deve ser descentralizada e contar com a participação do poder público, dos usuários e das comunidades.

Os estabelecimentos de ensino (universidades, escolas agrícolas, entre outros), ao se integrarem na gestão participativa dos recursos hídricos, são capazes de aprimorar a produção de estatísticas da água e coeficientes técnicos. Isto é possível pelo detalhamento da escala de mapeamento dos usos de recursos hídricos nos diversos setores institucionais (agropecuária, industria e famílias). Tal aprimoramento pode ser impulsionado pela adoção dos princípios da Educação Ambiental, que segundo Chung \& Vieira de Lima (2007) apud Reigada \& Reis (2004), é um processo educativo que visa formar cidadãos éticos nas suas relações com a sociedade e com a natureza. Estes autores destacam a interdisciplinaridade da Educação Ambiental como um catalisador para o conhecimento do meio ambiente.

Entre as disciplinas, a Estatística fornece insumos importantes para os estudos ambientais desenvolvidos em bacias hidrográficas, tais como:

- Definição do tipo de pesquisa (se amostral ou censitária);

- Definição do tamanho da amostra (se amostral),

- Direcionamento da amostra (de forma a contemplar a diversidade de usos da água)

- Elaboração de mapas estatísticos (utilizando análises multicritério, análises espaciais ou geoestatística).

\section{CONCLUSÕES}

A aplicação dos coeficientes técnicos de uso de recursos hídricos às estatísticas econômicas de produção industrial constitui em uma importante meio para a obtenção indireta de estatísticas ambientais. Entretanto algumas limitações para a aplicação direta destes coeficientes devem ser consideradas. Neste estudo de caso, os principais problemas encontrados foram:

- Significativa amplitude verificada em alguns coeficientes técnicos, talvez devido a diferenças de tecnologias aplicadas na produção dentro da mesma classe de atividade industrial; 
- Necessidade de conhecimento do peso específico ou densidade de alguns produtos industriais, não encontrados em pesquisas na Internet;

- Incerteza quanto adoção de uma medida-padrão para as matérias primas (gramatura, largura);

- A impossibilidade de mensuração dos recursos hídricos utilizados na produção de matériasprimas de consumo intermediário na produção industrial.

É importante pontuar que os resultados são referentes à aproximadamente $25 \%$ do total da produção industrial brasileira, integrante das estatísticas oficiais. A resolução dos problemas acima elencados pode ampliar o alcance dos coeficientes técnicos. Com relação à comparação dos resultados obtidos com dados primários, esta etapa não foi abordada neste estudo de caso. Entretanto, ao comparar os resultados com as estatísticas de estoque de recursos hídricos superficiais (armazenados em reservatórios), os resultados são qualitativos e subestimados.

O refinamento dos resultados obtidos pode ser possível por um ganho de escala na obtenção de estatísticas de uso de recursos hídricos pelos setores institucionais, no âmbito de bacias hidrográficas. Neste sentido, as instituições de ensino desempenham importante papel, por agregarem à análise questões tratadas na Educação Ambiental com base nos conceitos da Estatística, fundamental para os estudos objetivando a orientação do desenvolvimento sustentável local.

\section{REFERÊNCIAS BIBLIOGRÁFICAS}

Agência Nacional de Águas - ANA (2002). Manual de Procedimentos para Outorga de uso da Água na Indústria e Mineração. Brasília: ANA.

Agência Nacional de Águas - ANA (2012). Conjuntura dos Recursos Hídricos no Brasil - Informe 2012. Brasília: ANA.

BRASIL (1997). Lei Federal n 4.933 - Política Nacional de Recursos Hídricos.

Chung, F.G. \& Vieira de Lima, M.M. (2007). Educação Ambiental com Ênfase na Preservação dos Recursos Hídricos no Município de Campos do Jordão-SP. In Anais I Seminário de Recursos Hidricos da Bacia Hidrográfica do Paraíba do Sul: o Eucalipto e o Ciclo Hidrológico. Taubaté, Brasil: Instituto de Pesquisas Ambientais em Bacias Hidrográficas (pp. 131-137).

Fundação de Apoio à Universidade de Viçosa - FUNARBE (2011). Relatório Técnico 6 -

Relatório Final dos Coeficientes Técnicos de Recursos Hídricos das Atividades Industriais e Agricultura Irrigada - Desenvolvimento De Matriz De Coeficientes Técnicos Para Recursos Hídricos No Brasil. Brasília, 2011, 245 p.

Instituto Brasileiro de Geografia e Estatística - IBGE (2010a). Classificação Nacional de Atividades Econômicas Rev. 2.0 - CNAE. Rio de Janeiro: IBGE.

Instituto Brasileiro de Geografia e Estatística - IBGE (2010b). Pesquisa Anual da Indústria Produto. Rio de Janeiro: IBGE

Instituto Brasileiro de Geografia e Estatística - IBGE (2010c). Lista de Produtos da Indústria PRODLIST. Rio de Janeiro: IBGE.

Lisboa, L. (2010). Matriz de Coeficientes Técnicos de Recursos Hídricos para o Setor Industrial Brasileiro. Dissertação de mestrado. Universidade Federal de Viçosa - UFV. Viçosa: UFV, 81.

Lisboa, L. et al (2014). Matriz de Coeficientes Técnicos de Recursos Hídricos para o Setor Industrial Brasileiro. In Revista Brasileira de Recursos Hídricos - RBRH, 19(3), 327-337.

Reigada, C. \& Reis, M.F.C.T. (2004). Educação Ambiental para crianças no ambiente urbano: uma proposta de pesquisa-ação. In Ciência \& Educação, 10(2), 149-159. 